\title{
THE EFFECT OF SPACE CHARGE AND MAGNETIC FIELD ON THE INTERPRETATION OF BEAM BRIGHTNESS
}

\author{
J. K. Boyd \\ Lawrence Livermore National Laboratory \\ University of California \\ Livermore, CA 94550 \\ August 20, 1985 \\ UCID- -20585 \\ DE86 003498
}

ABSTRACT

\begin{abstract}
Beam brightness has been measured experimentally using collimators and emittance selectors. The acceptance of both of these devices must be known to jnfer a brightness measurement. A computer code has been written to determine the effect of space charge and magnetic field on the acceptance of these devices. The region of validity of analytic acceptance formulas is investigated.
\end{abstract}

Work performed jointly under the auspices of the U. S. Department of Energy by Lawrence Livermore Nationa 1 Laboratory under contract $W-7405-E N G-48$ and for the Department of Defense under Defense Advanced Research Projects Agency ARPA Order \#4395 Amendment \#31, monitored by Naval Surface Weapons Center under document \#N60927-85-P010001; and SOIO/BMD-ATC MIPR \#H3-RPD-53-A127; and SOIO/ NSWC document \#N60921-85-POW0002.

\section{DISCLAIMER}

This report was prepared as an account of work sponsored by an agency of the United States Government. Neither the United States Government nor any agency theroof, nor any of their employees, makes any warranty, express of implied, or assumes any legal liability or responsibility for the sceuracy, completeness, or usefulness of any information, apparatus, product, or process dictowed, or septesents that ju uec would not infringe privulely onaed rights. Refer. enoe hercin to eny specilie commerciel product, proses, of sevice by trade name, trademirk, manufacturer, or otberwise dose not seckeserily contitute or imply its eadorsentent, recom. mendation, of faroring by the United Stetes Gowernment or any agedsy thereof. The viems and opinions of authors expresued berein do pot necenarily slate or reflect these of the Unitod Stetes Goverameat of any reacy thereof. 


\section{INTRODUCTION}

Beam brightness has been measured experimentally using two diagnostics. The first diagnostic is a set of two plates with current limiting holes. This diagnostic is referred to as a collimator. The second diagnostic consists of a pipe tmmersed in an axial magnetic field and is generaliy designated an emittance selector. Several questions have arisen concerning the experimental determination of brightness using these diagnostics. Brightness is a measure of the current contained in a transverse phase space volume, $v_{4}$. Generally the experimental brightness is abtained by measuring current transmitted through a device of known $v_{4}$ or phase space volume acceptance. The $v_{4}$ or acceptance of the collimator and emittance selector has been calculated analytically by Sessler linder a number of assumptions. The analytic collimatar $v_{4}$ is based on the assumption of straighi line orbits in a field free region. The analytic emittance selector $v_{4} 15$ based on a device length greater than a cyclotron wavelength and an assumed uriform magnetic field. Under various experimental canditions one or more of these assumptions may be violated.

The BOB (B fleld effect $\underline{\text { On }}$ Brightness) computer code was initially written to study the change of collimator or emittance selector $v_{4}$ due to magnetic fields. Recentiy a space charge model has been added. BOB has been used to evaluate the collinator $v_{4}$ in the presence of a magnetic field. It has also been used to determine $v_{4}$ for cases of sufficient current such that space charge is an issue. BOB has calculated the emittance selector $v_{4}$ for 
non-uniform magnetic field and for magnetic fields such that the device length is less than a cyclotron waveiength. As an engineering consideration $B O B$ also calculates the axial position of phase space or current loss. This afds in the detemination of where cooling may be required.

In Section I the fleld models and equations solved by $80 \mathrm{~B}$ are discussed. In Section II and III, $V_{4}$ calculations are presented for a number of collimator and emittance selector cases. Conclusions are sumarized in section IV.

The collimator cases are relevant to recent ATA and HBTS brightness experiments. The issues are the change of $v_{4}$ from the analytic solution caused by space charge and focusing coil magnetic field.

The emittance selector cases relate to the $v_{4}$ changes of the anticipated Paladin experiment parameters at $42 \mathrm{MeV}$ and the operation of d dagnostic emittance selector at 3.4 MeV. 


\section{BoB Equations and Field Models}

The purpose of the BOB code is to calculate the acceptance or $v_{4}$ of a collimator or emittance selector. Various models are then available to relate current and $v_{4}$ to brightness. Thus knowledge of $v_{4}$ is crucial to deterinining beam quality.

\section{$\underline{v}_{4}$ Phase Space Acceptance}

A four space integral defines $V_{4}$.

$$
v_{4}=\int d x \int d y \int d x^{\prime} \int d y^{\prime}
$$

In Eq. (1) a prime denotes differentiation with respect to $z$. The limits of integration in Eq. (1) are determined by values of $x, y, x^{\prime}$ and $y^{\prime}$ which are accepted by a particular device. The BDB code always assumes axisymetry and thus one of the integrations in Eq. (1) can be eliminated.

$$
v_{4}=2 \pi \int r d r \int d x^{\prime} \int d y^{\prime}
$$

The devices under consideration operate with relativistic beams so the $z$ velocity 15 clase to the speed of light, $c$. Normalizing velocities to $c$ and the device radius to a characteristic radius a yields

$$
v_{4}=\frac{1}{2} \pi a^{2} \int d\left(\frac{r}{a}\right)^{2} \iint B_{z}^{-2} d B_{1}^{2} \mathbf{G} \phi
$$

where $\vec{B}=\vec{V} / c$ and $\phi$ is the angle between $B_{r}$ and $B_{\theta}$ at a particular radius. The scaling of the integrals in Eq. (3) is such that the radia? integration goes from 0 to 1 . Q varies from 0 to $2 \pi, B_{1}$ ranges from zero to 
$\left(1-\gamma^{-2}-B_{20}^{2}\right)^{1 / 2}$ and $B_{z}$ is approximately $1,\left(\gamma=\left(1-B^{2}\right)^{-3 / 2}\right)$.

The choice of $B_{20}$ is typically between .9 and 1 . Operationally BOB calculates Eq. (3) on a three-dimensional grid with axes of $(r / a)^{2}, B_{1}^{2}$ and $\phi$. Thus it is only necessary to sum up the inftial $\mathrm{B}_{\mathrm{z}}^{-2}$ values which are accepted.

$$
v_{4}=\frac{1}{2} a^{2} \Delta\left(\frac{r}{a}\right)^{2} \Delta \beta_{1}^{2} \Delta \phi \sum \beta_{2}^{-2}
$$

The $\Delta$ quantities in Eq. (4) are the mesh spacings. To determine if a particular phase space point is accepted it is necessary to follow an orbit using the $r, B_{1}$, $\phi$ phase space potnt as an initial condition.

\section{Equations of Motion}

The orbit is followed by solving equations for $x, y$. and $U=r B$. $A$ shifted and normalized 2 coordinate $s=(z-d) / L$ is used as the independent variable rather than time. The coordinate $s$ has vatues from 0 to 1 . The fields are normalized ty a characteristic magnetic fleld value $B_{D}$, and $x, y$ are normalized by the collifmator or emittance selector radius a. Taking the device length as $L$ the equations of motion are then,

$$
\begin{aligned}
& \frac{d x}{d s}=2 U_{x} / U_{z} \\
& \frac{d y}{d s}=2 \quad U_{y} / U_{z} \\
& \frac{d U}{d s}=\frac{x^{2}}{U_{z}} x_{c} L(\vec{E}+\vec{U} \times \vec{B} / Y)
\end{aligned}
$$

where $\dot{k}_{c}=q B_{0} /\left(r^{2} c^{2}\right.$ is a cycictron wave number and $\ell=L / a$. 


\section{Fleids}

The fields used in Eq. (5C) are models of coiis or space charge effects. Recent]y a $C_{4}$ magnet (solenoidai focusing coil) was placed in front of the callimater used to measure the brightness of the ATA accelerator. This coil is modeled as a single current loop of radius $r_{c 4}$ causing a field of strength $B_{0}$ at a distance on the entrance to the collimator. Because the collimator radius is small compared to the $C_{4}$ magnet radius the $B_{z}$ magnetic field component is assumed to be independent of radius. The normalized fjelds are below.

$$
\begin{aligned}
& B_{z}=\left(r_{c 4}^{2}+d^{2}\right)^{3 / 2} /\left(r_{c 4}^{2}+(s L+d)^{2}\right)^{3 / 2} \\
& B_{r}=\frac{3 a r}{2}(s L+d)\left(r_{c 4}^{2}+d^{2}\right) /\left(r_{c 4}+(s L+d)^{2}\right)^{5 / 2}
\end{aligned}
$$

The radial magnetic field in Eq. (6b) is consistent with the requirement $\nabla \cdot B=0$. The flelds in $\mathrm{Eq}$. (6) represent steady state magnetic fields in a source free region, thus $\nabla \times \vec{B}$ should be zera. Since this requirement is not satisfied a sma1l amount of non-physical current has been introduced. No adverse effects have been encountered in computing $v_{4}$ with $\nabla \times \vec{B}$ not equal zero. This is because the lead $B_{r}$ term for an expansion about the axis goes like $r$.

The magnetic field for the emittance selector is modeled as the fieid due to one or more current sheets. A particular current sheet has a radius $R$ and extends from $z_{1}$ to $z_{2}$. The $z$ component of the magnetic field is assumed to be the axial value multiplied by a radial profile factor which is approximately or.e . 


$$
\begin{gathered}
B_{z}=.5\left(1+a r^{2}\right)\left[\left(z_{2}-z\right) /\left(R^{2}+\left(z_{2}-z\right)^{2}\right)^{1 / 2}\right. \\
\left.+\left(z-z_{1}\right) /\left(R^{2}+\left(z-z_{1}\right)^{2}\right)^{1 / 2}\right] \\
B_{r}=.25 a\left(r+.5 a r^{3}\right)\left[\left(R^{2}+\left(z_{2}-z\right)^{2}\right)^{-1 / 2}\right. \\
\quad-\left(z_{2}-z^{2}\right)^{2} /\left(R^{2}+\left(z_{2}-z\right)^{2}\right)^{3 / 2} \\
\left.-\left(R^{2}+\left(z-z_{1}\right)^{2}\right)^{1 / 2}+\left(z-z_{1}\right)^{2} /\left(R^{2}+\left(z-z_{1}\right)^{2}\right)^{3 / 2}\right]
\end{gathered}
$$

The a parameter is a small number such as $1 / 100$ or $1 / 20$.

\section{Space Charge Fields}

The beam self fields arè abtained by assuming a uniform density beam with only a $z$ directed current. Solving $\nabla \times \vec{B}=4 \pi \vec{j} / c$, and $\nabla \cdot \vec{E}=4 \pi \rho$ yields,

$$
\begin{aligned}
& B_{\theta}=\frac{2 a_{z}^{2}}{c B_{0}}\left(\frac{I}{a^{2}}\right) a r \\
& E_{r}=r B_{\theta} / U_{z}
\end{aligned}
$$

where $a_{z}^{2}$ is only a function of $z$ which is related to the beam expansion be tween the collimator entrance and exit. To approximate various assumed beam expansion profiles BOB provides five different options.

$$
\begin{aligned}
& a_{z}^{2}=1 /\left(1+\left(\frac{z}{L}\right)^{2}\left(a_{f}^{2}-a^{2}\right) / a^{2}\right) \\
& a_{z}^{2}=a^{2} / a_{f}^{2}
\end{aligned}
$$




$$
\begin{aligned}
& a_{z}^{2}=\exp \left(-2 \frac{z}{L} \ln \left(a_{f} / a\right)\right) \\
& \left.a_{z}^{2}=1 /\left(1+\left(\frac{z}{L}\right)^{2}\left(a_{f}-a\right) / a\right)\right)^{2} \\
& a_{z}^{2}=1 /\left(1+\frac{z}{L}\left(a_{f}-a\right) / a\right)^{2}
\end{aligned}
$$

For Eq. $(9 a, c, d, e)$ the $a_{f}$ parameter is determined by assuming $a_{z}^{2}(L)$ is the ratic of exit to entrance current. In all cases $a_{f}$ is the radius at $z=L$. The motivation for the Eq. (9a) expansian profile is the canstant energy envelope equation solution with no space charge. The envelope equation solution gives $a^{2}(z)=a^{2}+z^{2} E^{2} / a^{2}$ which is of the same form as Eq. (9a). The Eq. $(9 b, c, d, e)$ expansion profiles correspond to radius variations for which analytic $V_{4}$ solutions can be derived [1]. These profiles are incorporated in $\mathrm{BOB}$ so they can be run with additional magnetic fields for which there is no analytic solution. BOB thus has the capability of simultaneously examining the effect of space charge and magnetic field. 


\section{Collimator $\underline{v}_{4}$ Calculations}

A collimator has been used to measure brightness on the ATA and HBTS experiments. Operationally the current through the first and second hole is measured. The brightness $F$ is def tned to be,

$$
g=\frac{\pi^{2} I_{2}}{(\gamma \beta)^{2} v_{4}}
$$

where $I_{2}$ is the current through the second hole. The acceptance for a pipe, assuming straight line orbits has been calculated by Sessier [2].

$$
v_{4 \text { Sessier }}=r^{2} a^{4} / L^{2}
$$

Using Eq. (11) in Eq. (10) the brightness can be expressed in terms of energy, current and geometric factors.

$$
\mathscr{J}_{\text {Sess Ter }}=\frac{L^{2} I_{2}}{(\gamma \beta)^{2} a^{4}}
$$

The brightness of the ATA and HBTS expariments have been calculated using Eq. (12). Due to the experimental environment the assumption of straight line orbits used to derive Eq. (12) is not vaTid for ATA or HBTS. There are two reasons for this difficulty. First the ATA experiment had a magnet near the collimatur. Second both 9TA and HBTS have a substantial deviation from straight line orbits due to space charge. 
Magnetic Field Effect on the Collimator Acceptance

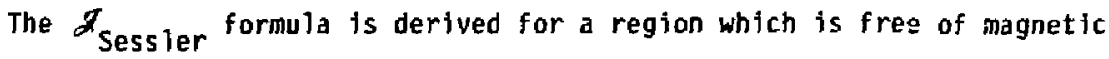
field. Recent measurements for a typical ATA tune show the collimator entrance is in a field of 100 gauss and the exit is in a field of 3 gauss.

The presence of a magnetic field causes orbit changes that result in exclusion of field free orbits which would otherwise be accepted. Likewise orbits whict would be excluded may be accepted. The effect is examined in two parts. First assume the region between the collimator entrance and exit has zera magnetic field but there is a non-zero field before the entrance. Write Eq. (1) in cylindrical coordinates, letting $v_{z}=c$.

$$
v_{4}=2 \pi c^{-2} \int r d r \int d v_{r} \int d v_{\theta}
$$

There are two constraints for acceptance of a phase space point.

$$
\begin{gathered}
r^{2} \leq a^{2} \\
\left(r+L v_{r} / c\right)^{2}+L^{2} v_{a}{ }^{2} / c^{2} \leq a^{2}
\end{gathered}
$$

The linits of integration are $C$ to a for $r$, and $-c(a+r) / L$ to $c(a-r) / L$ for $v_{r}$. The limits for $r$ come fram Eq. (14a) and the limits for $v_{r}$ come from Eq. (14b) with $v_{\theta}=0$. To obtain the limit of integration for $v_{\theta}$ it is necessary to consider the canonical momn ntum, since it is the conserved quantity.

$$
P_{\theta}=\gamma m r V_{\theta}+q r A_{\theta} / c
$$

From Eq. (15) in the field free case $v_{0}=P_{\theta} /(\gamma m r)$. The field free limits of integration from Eq. (14b) for $v_{\theta}$ are 


$$
\pm c\left(a^{2}-\left(r+L v_{r} / c\right)^{2}\right)^{1 / 2} / L
$$

Equation (16) gives the limits of integration for $\mathrm{P}_{\theta} /(\mathrm{mr})$. In the model beinu used this means the maximum $y_{\theta}$ value that is allowad at the collimator entrance is increased by $q A_{e} /(Y m c)$, and the minimum is increased by the same amount. In other wards the limits of integration are below.

$$
\begin{aligned}
& -c\left(a^{2}-\left(r+L v_{r} / c\right)^{2}\right)^{1 / \Omega} / L-a A_{\theta} /(r m c) \\
& c\left(a^{2}-\left(r+i v_{r} / c\right)^{2}\right)^{1 / 2} / L-a A_{\theta} /(r m c)
\end{aligned}
$$

Because the $v_{\theta}$ integrand in EG. (13) is unity the magnetis field effect subzracts out and $v_{4}$ is unchanged. This weans a group of particles excluded by their angle intercept of t le collimator entrance are accepted after they cross into the fleld free region ano thange their angle. Likewise an equal group of accepted particles are excluded after they cross into the field free region. Thus in the model of a very rapidly changing maguetic field $v_{4}$ is unchanged. The indicated integration in Eq. (4) yie]ds $\pi^{2} a^{4} / L^{2}$, which is the fleld free result. A more detailed argument with $\beta_{\overline{4}} ; 1$ can be made, however the conclusion is the same.

The primary reason for assuming a step function variation in magretic fieli was to make Eq. (14b) valid. The [q. (14b) restriction is derived assuming straight line orbits. The conclusion for this case is that there is no magnetic field effect on $V_{4}$. The next levei of complexity is the change of $v_{4}$ due to the deviation of orbits from straight lines caused by magnetic field 
between the collimator entrance and exit. In ather words a magnetic field between the collimator entrance and exit changes the Eq. (14b) constraint. For this case the constraint depends on the magnetic field in a complicated way and the 15sue must be examined numertcally. A useful figure of merit, $f_{m} t_{0}$ estimate the degree of acceptance change caused by the magnetic field is the integral of $k_{c}$.

$$
f_{m}=\int k_{c} d z
$$

For the fleld of Eq. (6a).

$$
\begin{aligned}
& f_{m}=k_{c 0}\left(1+\left(d / r_{c 4}\right)^{2}\right)^{j / 2} \int_{d}^{L}\left(1+\left(z / r_{c A}\right)^{2}\right)^{-3 / 2} d z
\end{aligned}
$$

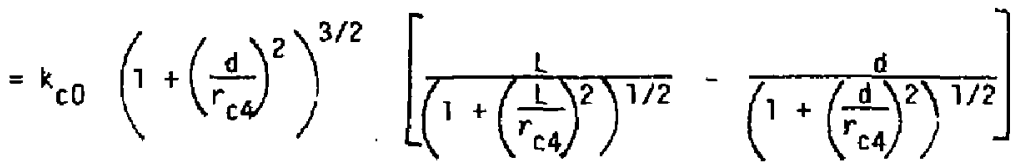

The BOB computer code has been run with no space charge using the Eq. (16) magnetic fleld. The paraineters in Tabie I are chosen to correspond to the ATA experiment.

\begin{tabular}{|lc|}
\hline$r_{\mathrm{c4}}$ & $8 \mathrm{~cm}$ \\
$d$ & $22 \mathrm{~cm}$ \\
$\mathrm{~L}$ & $3 \mathrm{~cm}$ \\
$r$ & 7 \\
$a$ & $.2 \mathrm{~cm}$ \\
\hline
\end{tabular}

Table I 
For these parameters $k_{c 0}=8.4 \times 10^{-5} \mathrm{~B}_{0}$ and $f_{m}=6.53 \times 10^{-4} B_{0}$. Cases were run with $B_{0}$ varying from 100 to 3000 gauss, and correspondingly $f_{m}$ varying from .0653 to 2 . The results are plotted in Fig. 1 . The abscissa is $L$ divided by the cyclotron wavelength at the colltmator entrance. This corresponds to $.5 \mathrm{~B}_{0} \mathrm{kgauss}$ and thus the abscissa is linear in the magnetic field. The ordinate is the ratio of the acceptance computed by BOB divided by the analytic field free acceptance. The plat shows the acceptance can increase from a few percent, to greater than $50 x$ at 3 kgauss. This means the $\mathscr{I}_{\text {Sessler }}$ brightness would have to be divided by 1.5 to account for the increased acceptance due to the magnetic field. For the field size of 100 gauss reported tu be present in the ATA experiment the magnetic field effect on $v_{4}$ is small.

Space Charge Effect on the collimator Acceptance

The presence of space charge causes the collimator acceptance to decrease. This occurs because the net beam expansion in the collimator results in the lass of particles which are accepted in the case of straight line orbits. The deviation from straight line orbits means $\mathscr{J}_{\text {Sessler }}$ under estimates the brightness. The true brightness is thus larger by a factor of $V_{4 \text { Sessler }} N_{A}$, where $v_{A}$ is the actual four space volume of the collimator.

The effect of space charge is modeled in the BOB code by the $B_{\theta}$ and $E_{r}$ fields of Eq. (B). In this model the net outward force per charge is $E_{r} / r^{2}$. This means the net outward force is always positive and the effect of space charge diminishes as energy increases. A consequence of this model is that a scaling factor of $\left(1 / a^{2}\right) a$ can be factored from the Eq. (5c) force equation. 
Assuming constant $Y, L$, and $a_{z}^{2}$ the nomalized $x, y$ coordinates only depend on $u_{x} / a$ and $u_{y} / a$ respectively. This means all solutions with the same $1 / a^{2}$ yield the same $v_{4}$. Equivalently the space charge effect scales with the current density at fixed energy and collimator length.

The BOB code has been run to determine the effect of space charge in the ATA and HBTS collimator. Because the BOB code was originally designed to examine magnetic fields, the force equation has a $k_{c}$ scale factor. To run space charge without external fieids it is necessary to retain a small finite value of $k_{c}$. For the ATA and HBTS collimator runs the magnetic field is set to $10^{-5}$ gauss. From results in the previous section a fleld of this size does nct have a significant effect on $V_{4}$.

The parameters in Table II correspuno to the ATA collimator and the HB. collimator.

\begin{tabular}{|l|c|c|}
\hline & ATA & HBTS \\
\hline$L(\mathrm{~cm})$ & 38 & 27.9 \\
$\mathrm{a} Z$ & Eq. (9a) & Eq. (9a) \\
$\mathrm{a}(\mathrm{cm})$ & .2 & .152 \\
$\mathrm{a}_{\mathrm{f}}(\mathrm{cm})$ & .4 & .45 \\
$\mathrm{Y}$ & 7 & 3 \\
\hline
\end{tabular}

Table II 
Cases were run for $1 / \mathrm{a}^{2}$ varying from 1250 to $12,500 \mathrm{amp} / \mathrm{cm}^{2}$. The results for ATA are platted in Flg. 2. The space charge effect is shown to be a monotont-

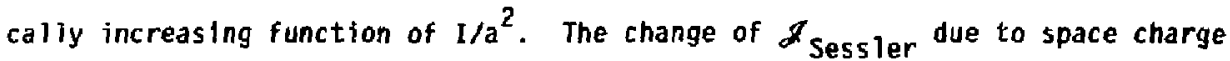
is obtained by multiplyting by $v_{4 \text { Sessler }} / v_{4}$. For example $1 / a^{2}=5000$ glves a brightness increase of $50 *$ or in other words $\mathscr{J}=1.5 \mathscr{Z}_{\text {Sess }}$ ler Recent ATA results [3] show operation of the collinatar with $2.6 \times 10^{3}<1 / \mathrm{a}^{2}<1 \times 10^{4}$ $\mathrm{amp} / \mathrm{cm}^{2}$. The corresponding brightness change is $1.2<\mathscr{J} / \mathscr{g}$ Sess ler $<2.4$. The best (brightest) ATA data with a hole radius of $a=.2 \mathrm{~cm}$ has $4.4 \times 10^{3}<1 / \mathrm{a}^{2}$ $<7.6 \times 10^{3} \mathrm{amp} / \mathrm{cm}^{2}$ and therefore $1.4<\mathscr{Z} / \mathscr{Z}_{\text {Sessler }}<2$.

The HBTS results are plotted in Fig. 3. Due to the lower energy the space charge effect is much stronger than for the ATA case just discussed. From fig. 3 it can be seen the space charge force is $s$ large very little current is transmitted for values of $\mathrm{I} / \mathrm{a}^{2}>3 \times 10^{3} \mathrm{amp} / \mathrm{cm}^{2}$. At $1 / a^{2}=5 \times 10^{3}, V_{4 \text { Sess } 1 \in r} / N_{4}$ is six times larger than values obtained for ATA in Fig. 2. Because space charge is such a large factor HBTS is typically run with $1.3 \times 10^{3}<1 / \mathrm{a}^{2}<3 \times 10^{3} \mathrm{amp} / \mathrm{cm}^{2}[4]$. The corresponding acceptance range is $1.8<v_{4 S e s 5] e r} / v_{4}<4.5$. Thus the $\mathscr{I}_{\text {Sessler }}$ formula substantial:y underestimates brightness. In all cases the HBTS brightness is a factor of 1.8 larger and in some cases a factor of 4 larger. 


\section{Emittance Selector $\underline{v}_{4}$ Calculations}

\section{ATA Emittance Selector}

The brightness of the ATA injector has been measured with an emittance selector. Using Eq. (10) with the acceptance formula [2]

$$
v_{4 \operatorname{mag}}=\pi^{2} a^{4} k_{c}^{2} / 6
$$

where $k_{c}$ is the cyclatron wave number, the brightness can be expressed in terms of transmitted current and magnetic field.

$$
\mathscr{Z}_{\text {mag }}=6 \mathrm{I} /\left(\gamma \beta \mathrm{a}^{2} \mathrm{k}_{\mathrm{c}}\right)^{2}
$$

The Eq. (19) formula was derived ignoring magretic field non-uлiformities space charge and assuming an emittance selector length greater than a cyclotron wavelength.

The $B D B$ code has been run to investigate the change of $v_{4}$ caused by the presence of space charge in the ATA emittance selector. At a nominal operating field of 1 kgauss the ATA emittance selector is 1.3 cyclotron wavelengths long, and thus this is not an issue. The effect of space charge is $i: 1$ ustrated in Fig. 4 where $V_{4 m a g} / V_{4}$ is platted as a function of the incident current for $r=7$ and $a=1 \mathrm{~cm}$. The two curves are for $a_{f}=a$ and $a_{f}=2 a$ at $z=200 \mathrm{~cm}$. The first case shows the greatest effect since space charge is present at the same strength at all $z$ positions. In the second case for radius expansion of a factor of two the space charge force is reduced by a factor of four. Cansistent with the reduction of the space charge force the $a_{f}=2 a$ curve is below the $a_{f}=a$ curve. The actual ATA experiment ran at a naminal current of $2 \mathrm{kA}$ and thus $1.5<V_{4 \mathrm{mag}} / V_{4}<1.7$ from Fig. 4. 


\section{Paladin Emittance Selector}

The Paladin emittance selector is designed to be installed at the end of the ATA accelerator. The beam energy at this position is 40 to $50 \mathrm{MeV}$ and consequently space charge has no effect on $v_{4}$. There are however three other issues. First at a mognetic field level appropriate to $g=2 \times 10^{5}$ at $1 \mathrm{kA}$ the emittance selector length of $372.5 \mathrm{~cm}$ is only $.75 \lambda_{\mathrm{c}}$. Since this violates one of the assumptions used to derive $v_{4 m a g}$ an immediate question is how much this changes the acceptance. Second the magnetic field is generated by solenoids which are separated by gaps. The presence of gaps causes a magnetic field non-uniformity. Depending on the size of the non-unifarmity the deviation from the uniform field, $V_{4 m a g}$ can be substantial. Thus the decerdence sf acceptance on gap size must be determined. Third the fact that an emittance selector excludes some velocities means a portion of the entering beam can spili before exfting. As an engineering consideration it is important to know where the current is lost.

\section{Emittance Selector Length and $\underline{v}_{4}$}

The effect of the emittance selector length on acceptance has been studied with BOB by calculating $V_{4}$ for magnetic fields from 250 to 6000 gauss. The results are plotted in $\mathrm{Fig} .5$ as a function of $L / \lambda_{C}$. An auxiliary abscissa label of magnetic fleld is also provided. The enittance selector parameters are $L=372.5 \mathrm{~cm}$ and $\mathrm{a}=.4 \mathrm{~cm}$. The analytic acceptance formula, valid for 
$L>\lambda_{c}$ scales as the square of the magnetic field. The ordinate of Fig. 5 is $v_{4}^{7 / 2}$ scaled so a $45^{\circ}$ ithe represents the analytic acceptance. The $B 0 B$ results plot on the $45^{\circ}$ line for $L>.8 \lambda_{c}$. This transition region corresponds to a magnetic field of roughly 2000 gauss. For $L<.8 \lambda_{C}$ Fig. 5 snows the calculated $v_{4}$ is greater than the analytic value. Asymptotically tise calculated $v_{4}$ approaches the acceptance of a field free pipe as the magnetic field is reduced to zera. For a brightness of $2 \times 10^{5} \mathrm{amp} /(\mathrm{rad}-\mathrm{crm})^{2}$ at $1 \mathrm{kA}$ the emittance selector operates at the point where the acceptance begins to deviate from the analytic $45^{\circ}$ ifne.

Solenoid Gap Size and $\underline{v}_{4}$

The planned Paladin emittance selector is designed to be imersed in a magnetic field generated by six solenolds each having a length of $60 \mathrm{~cm}$. The averall length is $372.5 \mathrm{~cm}$ and thus there are $2.5 \mathrm{~cm}$ gaps between each solenoid. BOB ran a number of cases for gap sizes from 0 to $9.7 \mathrm{~cm}$ between solenoids. The magnetic field for each case was 1827 gauss which corresponds to the $1 \mathrm{kA}, 2 \times 10^{5}$ brightness indicated on Fig. 5. The emittance selector length was held fixed at $372.5 \mathrm{~cm}$ and different gap spaces thus correspond to varyting the length of solenoids. Each solenoid was represented as two concentric current sheets at radti of 4.5 and $8.25 \mathrm{~cm}$. The results are plotted in Fig. 6 as a function of the gap spacu. The ordinate of fig. 6 is the percent deviation of the analytic acceptance from the calculated acceptance. At the destgned gap spacing of $2.5 \mathrm{~cm}$ the acceptance changes approximately $7 x$ froin the analytic value. For the largest gap of $9.7 \mathrm{~cm}$ the changa is $26 \%$. 


\section{Current Loss}

The position along the emittance selector where current is lost can impact engineering considerations such as cooling and material damage. The BOB code orbits phase space volumes through the emittance selector to calculate acceptance. During this process a recors is kept of where orbits impact the wall. A plot of phase space loss per $\mathrm{cm}$ thus gives an indication of where current spill occurs. In order to actually equate phase spaca loss to current lass an assumption of unjform current per phase space volume must be made. Within this assumption a loss of equal phase space volumes results in identical current losses. For magnetic fiejds ranging from 250 to 6000 gauss the current 1055 per cm is plotted versus distance in Fig. 7. The magnetic fieid is labeled on each of Fig. 7a to Fig. Th. The abscissa for each plot is distance along the emittance selector from 0 to $372.5 \mathrm{~cm}$. The ardinate for Fig. 7a to $\mathrm{Fig}$. 7h is current loss in arbitrary units. The actual current loss in amp/cm can only be determined if the current contained in a jperific phase space volume is known. For particular choices of beam brightness and emittance selector magnetic field Fig. 5 may be used to ascertain the value or $V_{4}$. A simple application of the definition of brightness then yields the corresfonding current. The main use of $\mathrm{Fig} .7$ is in the interpretation of experimental data at low repetition rates. A measurement of current in and current transmitted through the emittance selector yields the current lost. The plots in fig. 7 then indicate the I position where the current loss occurs for particular magnetic flelds. Al estimate can thus be made of cooling requirements and possible material damage thresholds. These calculations can then be applied 
to high repetition rates to ensure successful operation. The plots in Fig. 7 Indicate current loss preferentially occurs at large $z$ for fields of, 25 and .5 kgauss. The loss is approximately uniform in $z$ at 1 kgauss. For magnetic fjelds of $2,3,4,5$ and 6 kgauss, the current loss is preferentially at small $z$ values. A double peak loss curve develops for magnetic fields between 2 and 6 kgauss. As the field strength increases the two peaks tend to coalesce. The general trend indicated by $\mathrm{Fig}$. 7d to $\mathrm{Fig}$. Th is that the current loss occurs at smaller and smaller $z$ positions as the magnetfc field increases. Since the acceptance or $v_{4}$ of the device incrases with increasing magnetic field the current which is lost at high magnetic field has very poor emittance. Thus, although the cuirent loss is concentrated over a small region the amount of current lost should be negligible in this case. Typically upstream of the enittance selector there are other beamline elements which filter out the partion of the beam current with large values of emittance. 


\section{Summary and Conclusions}

The $\mathrm{BOB}$ computer code has been writter to cajculate collimator and emittance selector acceptances. The integral definition of acceptance is evaluated by following phase space volume orbtts. Models have been incorporated to account for the effect of space charge and magnetic field variations on collimator and emittance selector acceptance. The space charge model is derived from the fields due to a beam of untform charge density cross section which has a radius that varies in 2 . Magnetic field options are provided for a focus magnet and axisymetric solenoids.

kesults have beer presented which impact the interpretation of beam quality in the ATA and HBTS experiments. Usuaily the acceptance of a collimator or emittance selertor is assumed to be given by analytic formulas which have been discussed. In the case of ATA and HBTS there are substantial deviations from the analytic acceptance values. The deviations are caused by the proximity of a focus magnet to the ATA collimator and the impact of space charge on the assumption of straight line orbits in the collimator and emittance selector. It was shown the magnetic field leading up to the ATA collimator is not important. It is the magnetic field which threads the collimator which impacts the acceptance. The figure of merit $f_{m}$ determines.. when this magnetic fleld effects acceptance. It was found under standard ATA operating conditions the magnetic field effect on acceptance is small. The effect of space charge results in a $20 \%$ to $200 \%$ changa in the expected ATA colltmator acceptance. For recent HBTS experiments space charge causes an $80 \%$ to $400 \%$ effect. 
Four emittance selector issues were investigated. It was found the low energy ATA emittance selector acceptance varied from $50 \%$ to $70 \%$ from the analytic acceptance due to space charge effects.

The other three issues are related to the Palavin emittance selector which operates at high energy in ATA. At high energy space charge effects are negligible. It wa, found for expected operating conditions the paladin emitrance selector is not quite a cyclotron wavelength long. Thus to assist in the understanding of the device an operating curve of acceptance versus megnetic field was calculated. The operating curve clearly shows the magnetic field values where the analytic acceptance formula is valid.

The Paladin emittance selector is designed with gaps between sulenoids which generate the magnetic fjeld. These gaps cause field non-uniformities which affect the acceptance. The gos code was used to calculate a curve of acceptance versus gap size. For the design gap size of $2.5 \mathrm{~cm}$ the acceptance changes by approximately $7 \%$ from the analytic value.

In the expected mode of operation not all the current entering the paladin emittance selector is transmitted. The current which is lost can preferentially split on various portions of the emittance selector depending an the strength of the magnetic field. Bab calculated severat cases to 113ustrate the current loss dependence on field strength. At low field values the current spills at large $z$ values near the exit. At high field values the current loss is concentrated at small z values near the emittance selector entrance. 


\section{REFEREMCES}

[1] G. J. Caporaso, "The Effects of Space Charge on the Acceptance of Brightness Measuring Devices," LLNL Report UCID-20527 dated Aug 14, 1985.

[2] A. M. Sessler, unpublished notes.

[3] 3. T. Weir and the ATA Staff, private communication.

[4] a. L. Birx and the HBTS Staff, private communication.

JKE:jna

0103a 


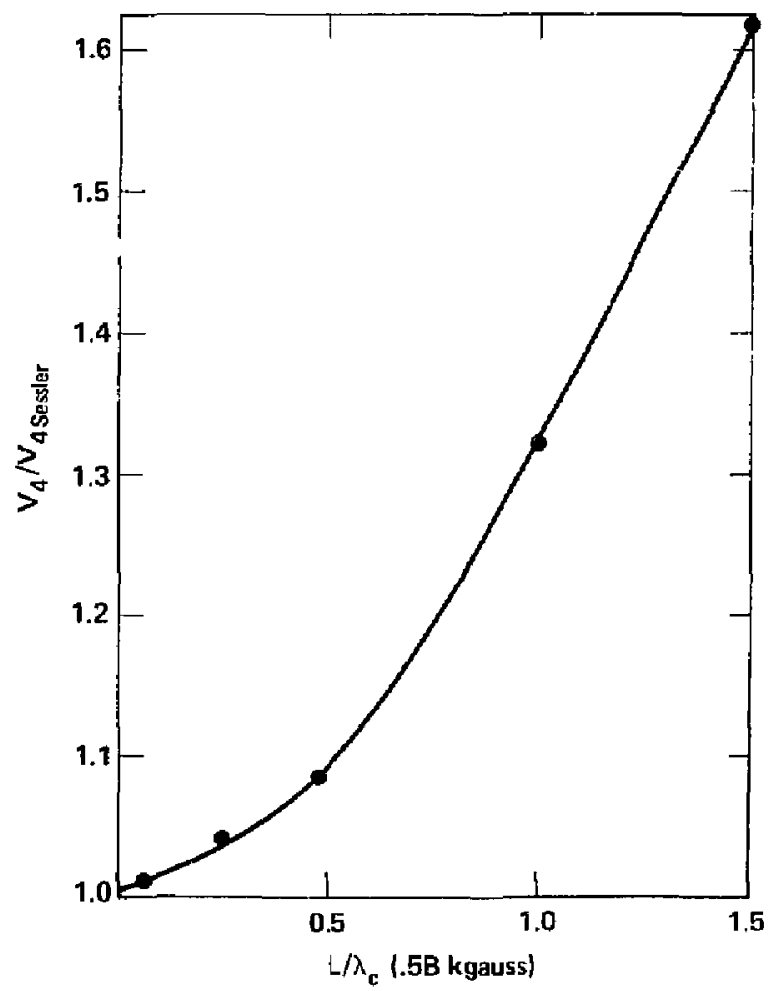

Figure 1. The normalized ATA collimator acceptance in the presence of a magnetic field is plotted as a function of inverse cyclotron wavelength. The abscissa corresponds to half the magnetic field value in units of kgauss. 


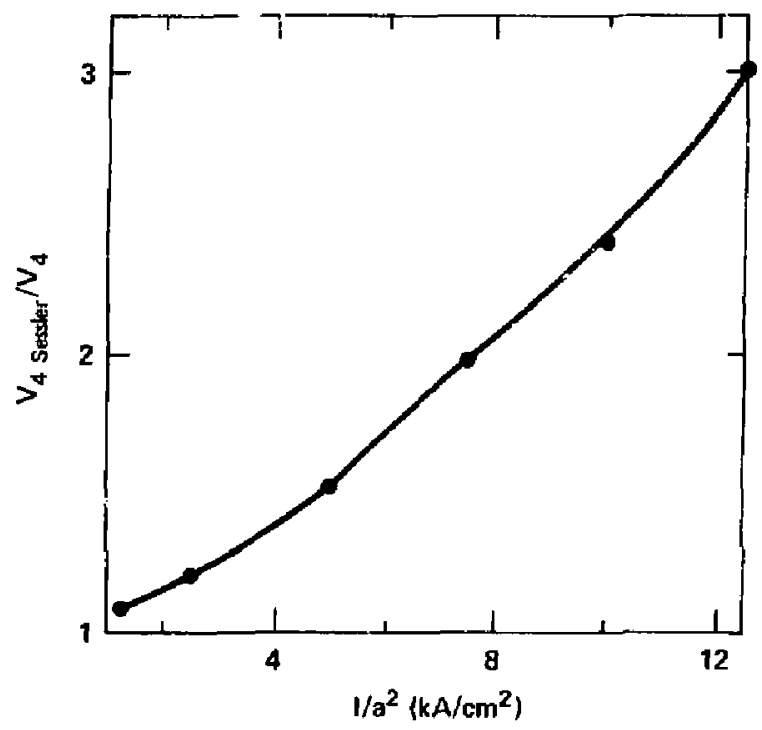

Figure 2. The inverse normalized ATA collimator acceptance is plotted as a function of space charge. 


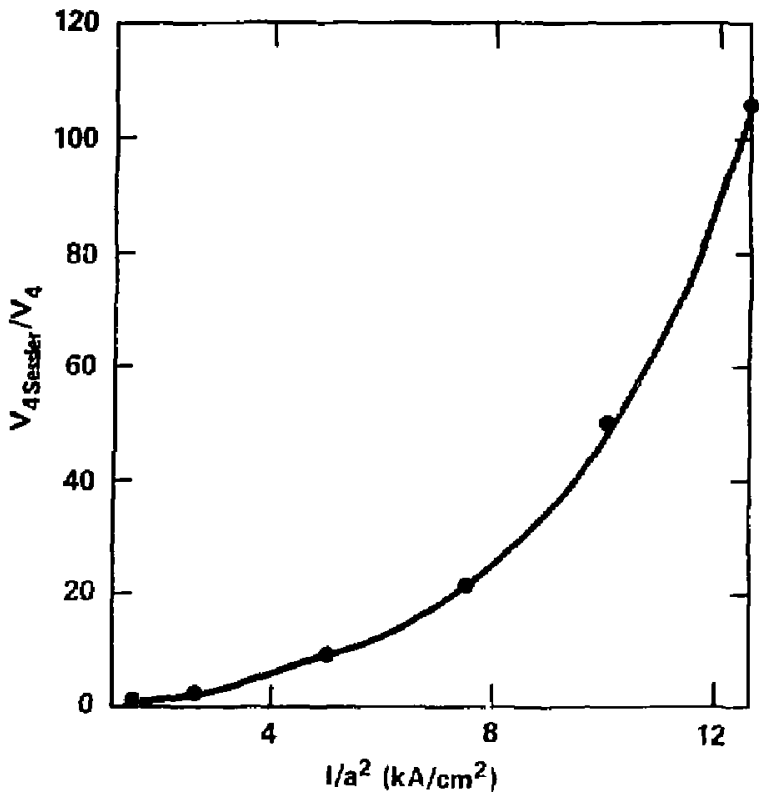

Figure 3. The inverse normalized HBTS collimator acceptance is plotted as a function of space charge. 


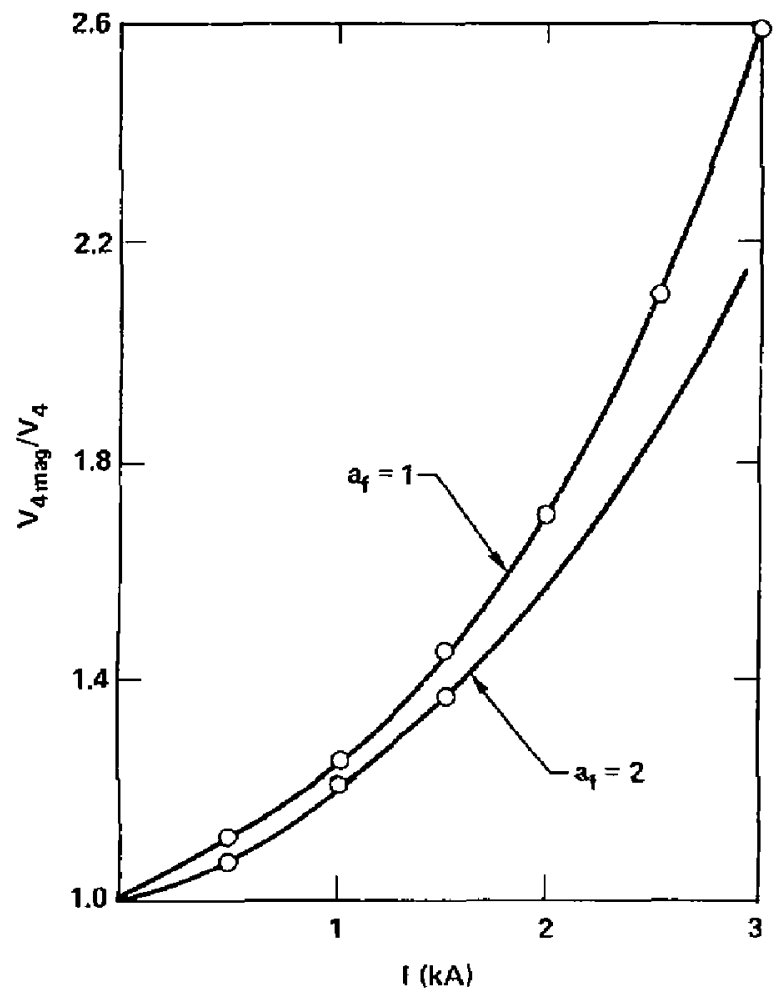

Figure 4. The effect of space charge on the ATA emittance selector acceptance is plotted for two beam expansion scenarios. 


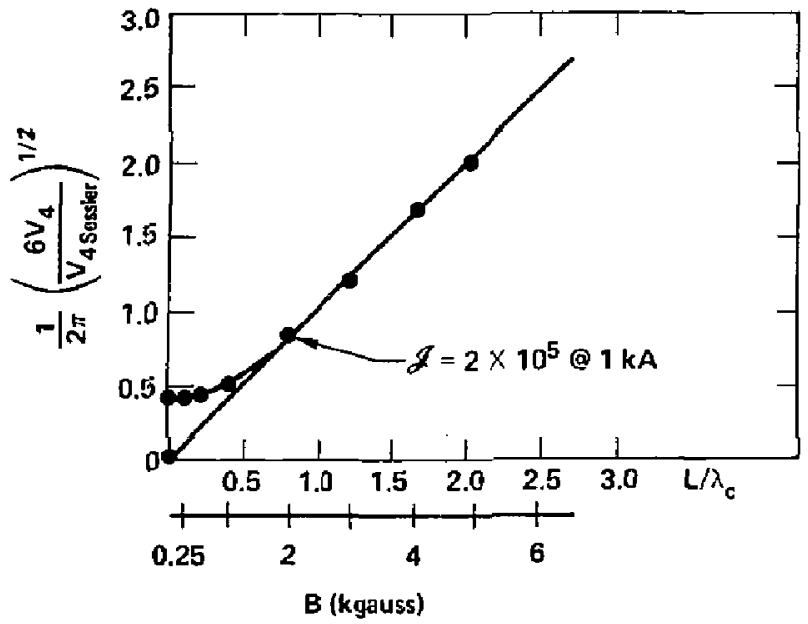

Figure 5. The Paladin emittance selector operating curve is plotted as a function of magnetic field or inverse cyclotron wavelength. The $45^{\circ}$ line corresponds to the analytic acceptance formula. 


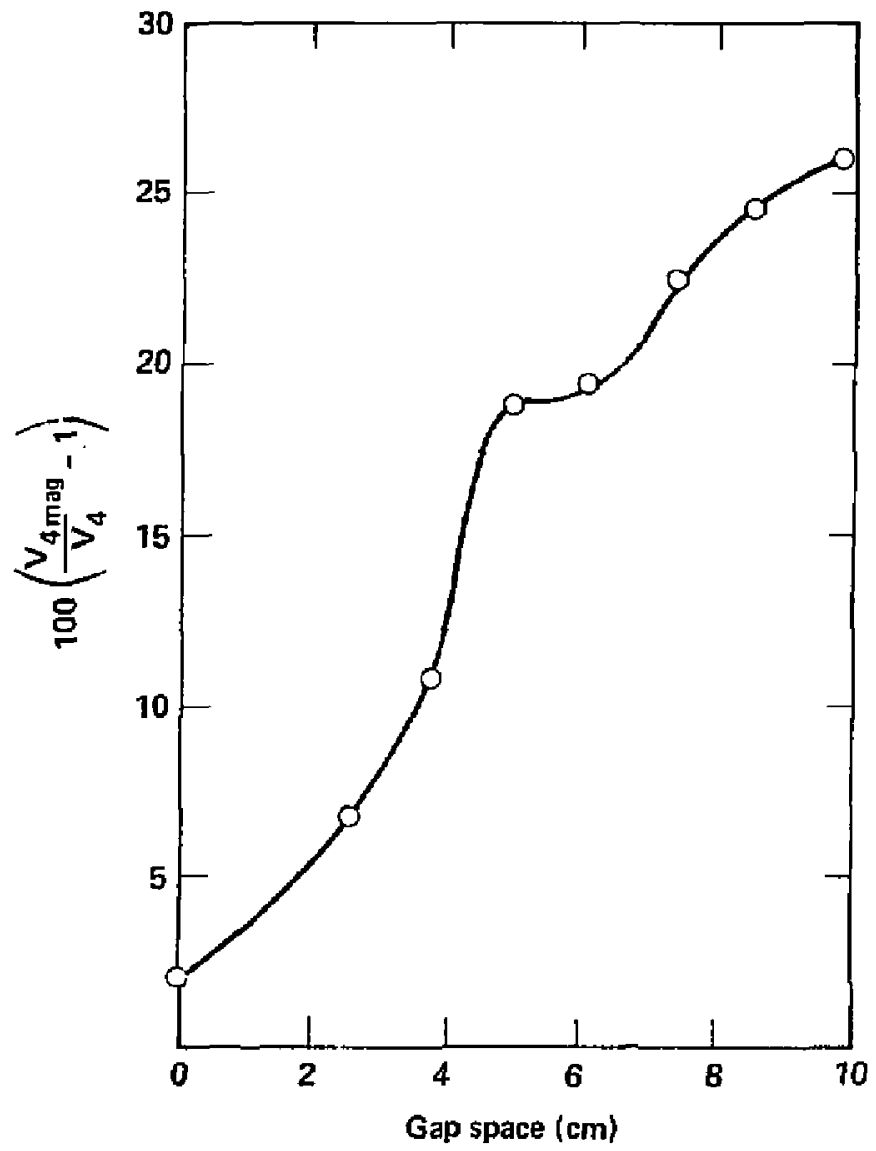

Figure 6. The percent change of the Paladin emittance selector inverse normalized acceptance is plotted as a function of solenoid gap size for a magnetic field of 1827 gauss. 

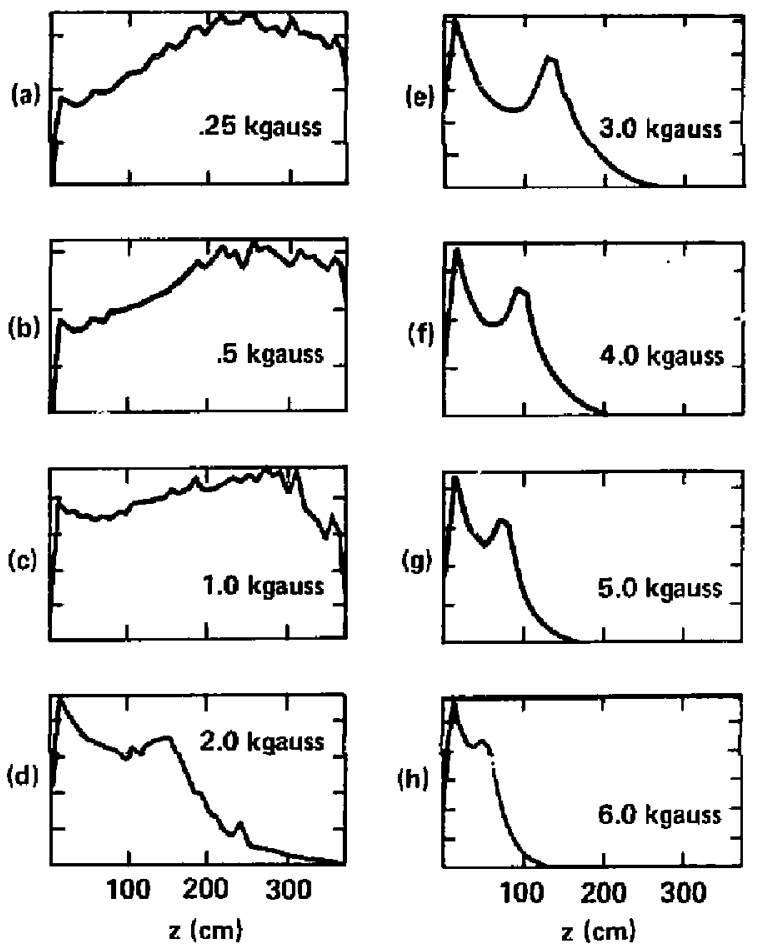

Figure 7. The Paladin emittance selector, axial current loss per $\mathrm{cm}$ profile is plotted for magnetic field values from . 25 to 6 kgauss. 